\title{
White Noise Characterization of N-MOSFETs for Physics- Based Cryogenic Device Modeling
}

This paper was downloaded from TechRxiv (https://www.techrxiv.org).

\section{LICENSE}

CC BY 4.0

SUBMISSION DATE / POSTED DATE

05-01-2021 / 07-01-2021

\section{CITATION}

Ohmori, Kenji; Amakawa, Shuhei (2021): White Noise Characterization of N-MOSFETs for Physics-Based Cryogenic Device Modeling. TechRxiv. Preprint. https://doi.org/10.36227/techrxiv.13520612.v1

$\mathrm{DOI}$

10.36227/techrxiv.13520612.v1 


\title{
White Noise Characterization of N-MOSFETs for Physics-Based Cryogenic Device Modeling
}

\author{
K. Ohmori and S. Amakawa \\ Device Lab Inc., Tsukuba, Ibaraki, Japan; Email: ohmori@devicelab.co.jp
}

\begin{abstract}
We propose a methodology of variable-temperature broadband noise characterization for cryogenic MOSFETs. A DUT is mounted on a reusable PCB vehicle with a built-in low-noise amplifier, and loaded into a cryogenic chamber. Using the vehicle, we measured flicker (low frequency) and white noise, and have successfully revealed dominance of shot noise in the temperature range from 300 to $120 \mathrm{~K}$ for the first time.
\end{abstract}

(Keywords: cryogenic CMOS, white noise, 1/f noise)

\section{Introduction}

Quantum computing is one of the challenging technologies that require the power of CMOS circuits be made available at cryogenic temperatures for maintaining the coherent time of qubits, such as electronic spin. A peripheral circuit for the control and readout of a quantum processor may be needed to operate at a temperature as low as 1 to $4 \mathrm{~K}$ [1]. Therefore, MOSFET compact models valid down to cryogenic temperatures are necessary [2]. At cryogenic temperatures, peculiar phenomena such as bandgap widening, carrier freezeout, and self-biasing appear. Continuous characterization of MOSFETs at varying temperatures starting from room temperature (RT) down to cryogenic temperature is important for building physics-based predictive models. In particular, wideband noise modeling is essential for the development of low-noise circuits interfacing with a quantum processor.

MOSFETs are known to exhibit flicker noise at low frequencies (LF) and white noise at high frequencies (HF). Physical origins of flicker noise and white noise differ, and therefore, their power spectral densities are independent of each other. White noise can be further classified into thermal noise and shot noise. Unlike the relationship between flicker noise and white noise, thermal noise and shot noise are not completely independent of each other [3-4].

A proof-of-concept noise probe for on-wafer broadband characterization was demonstrated using a dc-biased approach at RT, achieving HF noise measurement up to $800 \mathrm{MHz}$ [5]. At present, an improved version of the noise probe is commercially available as Entrope ${ }^{\circledR}$ Noise Probe [6], details of which will be presented elsewhere [7]. In this study, we have applied the noise probe technology to an insitu system of variable-temperature broadband noise measurements down to $120 \mathrm{~K}$.

\section{Experimental Setup and Measurement}

We developed a reusable PCB vehicle for cryogenic measurements, which includes a broadband lownoise amplifier (LNA). Each vehicle is characterized and calibrated using our proprietary technology so that we obtain pure noise from a DUT without noise from the LNA. A DUT is mounted on the vehicle right next to the LNA. We employed a semiconductor device analyzer (B1500A, Agilent Technologies) for biasing DUTs and a spectrum analyzer (N9030A, Agilent Technologies) for reading the output from the LNA.

We characterized two types of N-MOSFETs, denoted by $\mathrm{A}$ and $\mathrm{B}$, for demonstration of several kinds of noise characterization. The gate lengths are $240 \mathrm{~nm}$ for A and $120 \mathrm{~nm}$ for B. DUTs with 4 contact pads were mounted on vehicles by flip-chip bonding for stable measurements at different temperatures.

\section{Variable-Temperature Characterization}

\section{A. Instrumental Setup and Dc Properties}

The PCB vehicle is inserted in a cryogenic He chamber as schematically shown in Fig. 1. The temperature $(T)$ range examined is from 5 to $300 \mathrm{~K}$. If one uses a cryogenic probe station, the length of probe tips shrinks as $T$ decreases, which makes stable continuous variable- $T$ measurement very difficult. Therefore, it is beneficial also in this regard to bond a DUT next to an LNA.

Figure 2(a) shows $I_{\mathrm{d}}-V_{\mathrm{g}}$ curves at 5-300 K. At low temperatures, $I_{\mathrm{d}}$ decreases in the subthreshold region and increases in the strong inversion region corresponding to the change in the transconductance $\left(g_{\mathrm{m}}\right)$ shown in Fig. 2(b). Dependences of $V_{\text {th }}$ and subthreshold swing (SS) on $T$ are plotted in Fig. 3. SS becomes steep as $T$ decreases. The $V_{\text {th }}$ values shifts higher as $T$ decreases. We used the gatesource voltage $\left(V_{\mathrm{g}}\right)$ as biasing points in variabletemperature noise measurements instead of the gate overdrive voltage.

\section{B. Pure Thermal Noise at Zero Drain Voltage}

What is unique in this study using the vehicle is a capability of wideband noise characterization. Figure 4 shows $S_{\text {Id }}$ under a zero-drain-bias $\left(V_{\mathrm{d}}=\right.$ $0 \mathrm{~V})$ condition for $V_{\mathrm{g}}$ from 0.8 to $1.6 \mathrm{~V}$, demonstrating successful measurement of white noise at (a) $T=300$ and (b) $121 \mathrm{~K}$. The frequency range is from $50 \mathrm{kHz}$ to $100 \mathrm{MHz}$. Note that the maximum 
measurable frequency in commercially available LF noise systems is a few tens of $\mathrm{MHz}$ at most. As $V_{\mathrm{g}}$ increases, the differential resistance $R_{\text {dif }}\left(=\partial V_{\mathrm{d}} /\right.$ $\left.\partial I_{\mathrm{d}}\right)$ decreases, resulting in higher white noise levels.

Noise measurements were conducted continuously from 120 to $300 \mathrm{~K}$. In Fig. 5, $T$ dependence of $S_{\text {Id }}$ at $10 \mathrm{MHz}$ are plotted as a function of thermal noise intensity $S_{\text {th }}\left(=4 k_{\mathrm{B}} T / R_{\text {dif }}\right)$, where $k_{\mathrm{B}}$ is the Boltzmann constant. We observed a good linear relationship with the slope value of unity. As $V_{\mathrm{g}}$ and $T$ change, the range of the linear relationship extends from $4 \times 10^{-25}$ to $7 \times 10^{-23} \mathrm{~A}^{2} / \mathrm{Hz}$, which corresponds to the $R_{\text {dif }}$ values from $23 \mathrm{k} \Omega$ to $188 \Omega$.

\section{Shot-Noise Evaluation in the Linear Region}

When a nonzero $V_{\mathrm{d}}$ is applied, $I_{\mathrm{d}}$ starts to increase and shot noise appears. In order to characterize white noise that consist of thermal and shot noise, noise measurement was conducted in the linear region for DUT A, where $V_{\mathrm{d}}$ ranges from 0 to $0.08 \mathrm{~V}$ as shown in Fig. 6. The change from flicker noise to white noise is clearly observed. We focus on white noise characterization in this study. Although the slope of $S_{\text {Id }}(f)$ becomes very small at high frequencies, the slope for large $V_{\mathrm{d}}$ values is not quite zero. Therefore, we estimated $f_{\mathrm{c}}$ and $S_{\mathrm{w}}$ by using

$$
S_{\text {Id }}(f)=K_{\mathrm{f}} / f^{\beta}+S_{\mathrm{w}},
$$

where $K_{\mathrm{f}}$ is $S_{\mathrm{Id}}$ at $1 \mathrm{~Hz},-\beta(\approx-1)$ is the slope of flicker noise power spectrum on a log-log plot, and $\left(K_{\mathrm{f}} / S_{\mathrm{w}}\right)^{1 / \beta}$ corresponds to the corner frequency $\left(f_{\mathrm{c}}\right)$. Extracted $\beta$ and $S_{\text {Id }}$ values at 10 and $30 \mathrm{MHz}$ were used. As shown in Fig. 7, $f_{\mathrm{c}}$ gradually increases as $T$ decreases mainly because the intensity of thermal noise decreases. The $f_{\mathrm{c}}$ value is higher for larger $V_{\mathrm{d}}$ due to higher LF noise intensity.

Figure 8 shows the dependence of $S_{\text {Id }}$ and estimated $S_{\mathrm{w}}$ on $V_{\mathrm{d}}$ for $T=$ (a) 300 and (b) $130 \mathrm{~K}$. Open circles and diamonds show measured $S_{\text {Id }}$ at 10 and $30 \mathrm{MHz}$, respectively. Difference between these values becomes larger as $V_{\mathrm{d}}$ increase resulting from the increase in residual flicker noise. Thermal noise intensity $S_{\text {th }}$ for nonzero-drain-bias conditions is given by [8]

$$
S_{\text {th }}=4 k_{\mathrm{B}} T R_{\mathrm{ch}}\left(V_{\mathrm{d}}\right) /\left[R_{\mathrm{dif}}\left(V_{\mathrm{d}}\right)\right]^{2},
$$

where $R_{\mathrm{ch}}=V_{\mathrm{d}} / I_{\mathrm{d}}$ is the chord resistance [9].

Expected thermal noise $S_{\text {th }}$, full-shot noise $2 q I_{\mathrm{d}}$, and the sum of them are superimposed by the dashed, solid, and dot-dashed lines, respectively. At $V_{\mathrm{d}}=$ $0 \mathrm{~V}$, the $S_{\mathrm{w}}$ value corresponds to thermal noise $S_{\text {th }}$ and, as $V_{\mathrm{d}}$ increases, it increases along $2 q I_{\mathrm{d}}$. Shot noise is often characterized by using the so-called Fano factor and the $2 q I_{\mathrm{d}}$ value iss the maximum limit of shot noise [10]. Thermal noise dominates under low drain bias $\left(V_{\mathrm{d}} \lesssim 2 k_{\mathrm{B}} T / q\right)$ and shot noise becomes dominant when $V_{\mathrm{d}} \gtrsim 2 k_{\mathrm{B}} T / q$, which is indicated by the red arrows.

Figure 9 shows correlation between $S_{\mathrm{w}}$ and thermal noise $S_{\text {th }}$ for $120 \mathrm{~K} \leq T \leq 300 \mathrm{~K}$. As $T$ decreases, $S_{\mathrm{W}}$ at $V_{\mathrm{d}}=0 \mathrm{~V}$ (black circles) decreases exhibiting a linear relationship with the slope value of unity as also seen in Fig. 5, whereas $S_{\mathrm{w}}$ at $V_{\mathrm{d}}>0 \mathrm{~V}$ remains higher than the thermal noise, clearly demonstrating that shot noise becomes predominant at low temperatures. We believe that detailed description of this behavior using the Fano factor [7], for example, will provide predictive noise modeling at cryogenic temperatures.

\section{Conclusion}

We have demonstrated variable-temperature characterization of N-MOSFETs, using the precisely calibrated PCB vehicle for broadband noise measurements from flicker noise to white noise. In order to build physics-based predictive models for developed MOSFETs, actual temperature-dependent measurements of broadband noise are necessary. Our measurement data show that a cryogenic environment reduces white noise at $V_{\mathrm{d}}=0 \mathrm{~V}$, but does not reduce $1 / f$ and shot noise at $V_{\mathrm{d}} \neq 0 \mathrm{~V}$, which will be a problematic noise source for quantum computing.

The lowest temperature for noise characterization was limited to $120 \mathrm{~K}$ in this study because bipolar transistors were adopted for the LNA. We believe that our methodology proposed here can be extended down to $4 \mathrm{~K}$ or below by designing an LNA composed of MOS transistors.

\section{Acknowledgments}

The authors gratefully acknowledge the cooperative research with Prof. R. Hasunuma of University of Tsukuba.

\section{References}

[1] F. Sebastiano, et al., Proceedings of the 54th Annual Design Automation Conference 2017.

[2] A. Beckers, F. Jazaeri, and C. Enz, IEEE. Electron Device Lett. 41, 276 (2020).

[3] R. Landauer, Phys. Rev. B 47, 16427 (1993).

[4] Y. Tsividis and C. McAndrew, Operation and Modeling of the MOS Transistor, 3rd edition (Oxford University Press, 2011).

[5] K. Ohmori, et al., 2013 Symposium on VLSI Circuits Digest of Technical Papers, C40.

[6] http://www.devicelab.co.jp

[7] K. Ohmori and S. Amakawa, submitted.

[8] A. van der Ziel, Noise in Solid State Devices and Circuits (John Wiley \& Sons, Inc., New York 1986).

[9] L. Chua, Appl. Phys. A, 102, 765 (2011).

[10] C. Beenakker and C. Schönenberger, Phys. Today 56, 37 (2003). 


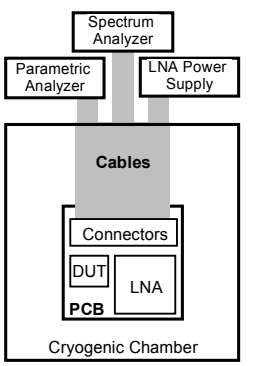

Fig. 1. Schematic of a cryogenic system with a PCB vehicle for wideband noise measurement.
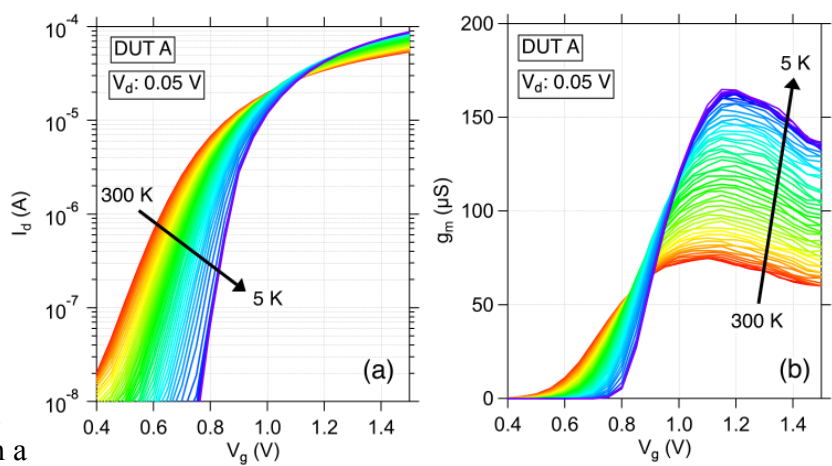

Fig. 2. (a) Typical $I_{d} V_{g}$ property and (b) transconductance $\mathrm{g}_{\mathrm{m}}$ for $\mathrm{T}$ ranging from 5 to $300 \mathrm{~K}$.
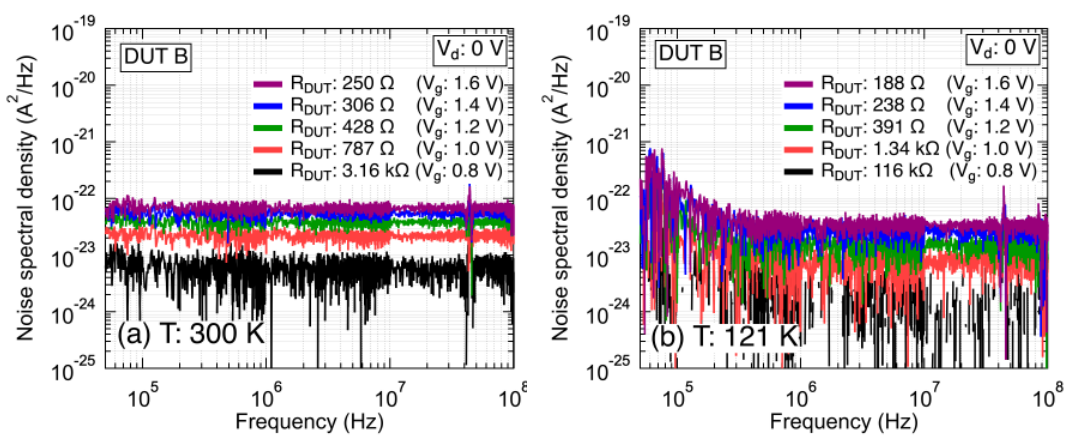

Fig. 4. Noise spectral density obtained at $\mathrm{V}_{\mathrm{d}}=0 \mathrm{~V}$ and at $\mathrm{T}=$ (a) $300 \mathrm{~K}$ and

(b) $121 \mathrm{~K}$. Under the same bias condition, white noise at $\mathrm{T}=121 \mathrm{~K}$ is smaller than that at $\mathrm{T}=300 \mathrm{~K}$.
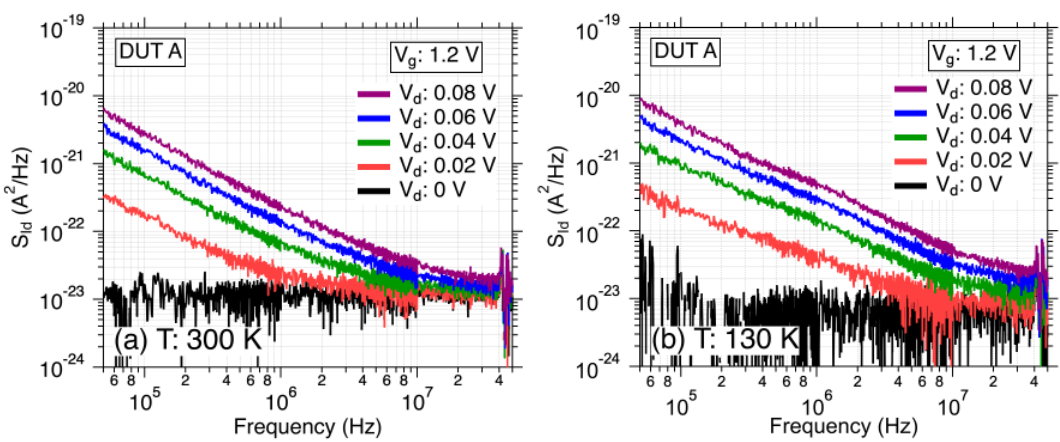

Fig. 6. Noise spectral density obtained at $\mathrm{V}_{\mathrm{g}}=1.2 \mathrm{~V}$ and at $\mathrm{T}=$ (a) $300 \mathrm{~K}$ and (b) $130 \mathrm{~K}$. As $\mathrm{V}_{\mathrm{d}}$ increases, $1 / \mathrm{f}$ noise appears and white noise increases.
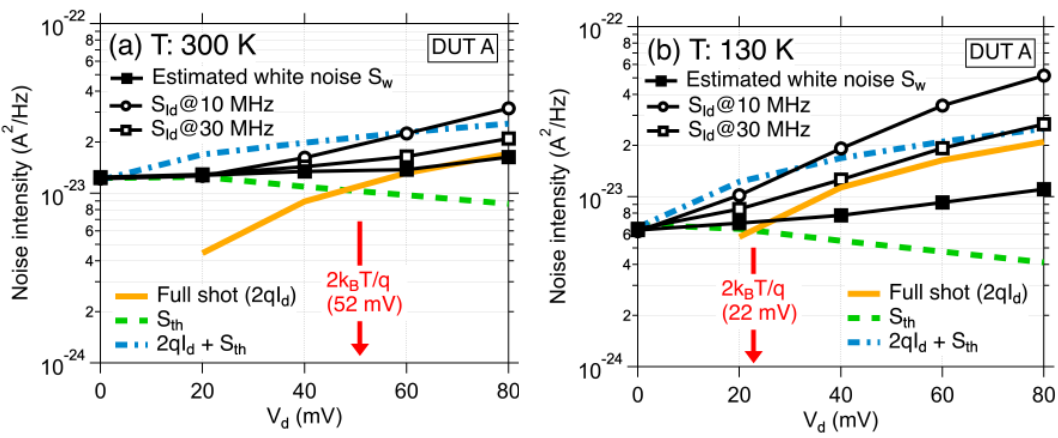

Fig. 8. Dependences of $S_{I d}$ on $V_{d}$ for $V_{g}=1.2 \mathrm{~V}$ at $T=$ (a) $300 \mathrm{~K}$ and (b) $130 \mathrm{~K}$. The intensity of estimated white noise is between $\mathrm{S}_{\text {th }}$ and $2 \mathrm{qI}_{\mathrm{d}}+\mathrm{S}_{\mathrm{th}}$. The $\mathrm{V}_{\mathrm{d}}$ value at which $2 \mathrm{qI}_{\mathrm{d}}=\mathrm{S}_{\mathrm{th}}$ is determined by $2 \mathrm{k}_{\mathrm{B}} \mathrm{T} / \mathrm{q}$ at each $\mathrm{T}$, where the increase of $S_{w}$ is actually observed.

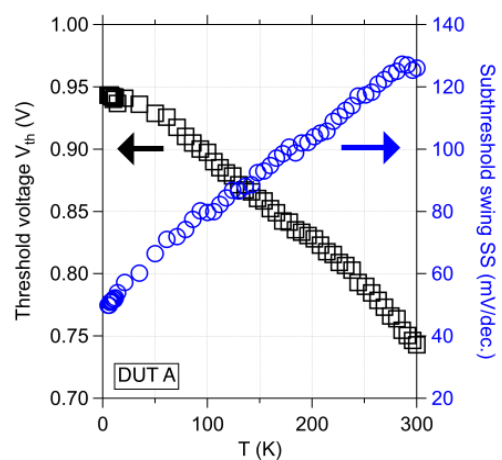

Fig. 3. $V_{\text {th }}$ and $\mathrm{SS}$ as a function of $\mathrm{T}$.

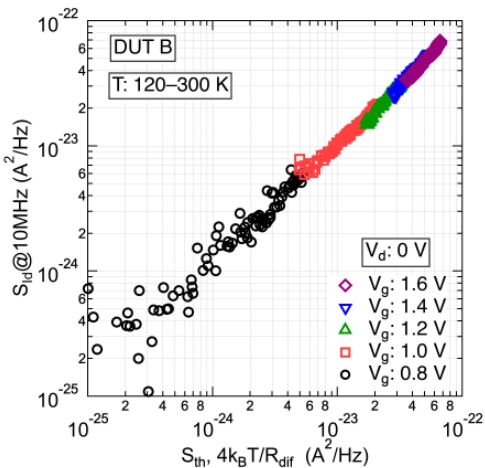

Fig. 5. Correlation between $S_{I d}$ and thermal noise $\left(4 \mathrm{k}_{\mathrm{B}} \mathrm{T} / \mathrm{R}_{\mathrm{dif}}\right)$ at $\mathrm{V}_{\mathrm{d}}=0 \mathrm{~V}$ for $\mathrm{V}_{\mathrm{g}}$ ranging from 0.8 to $1.6 \mathrm{~V}$.

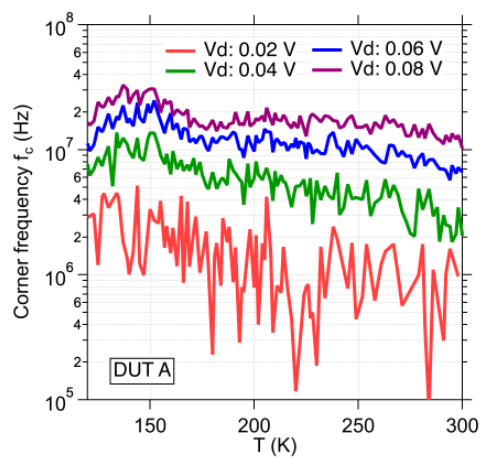

Fig. 7. Corner frequency $f_{c}$ as a function of $\mathrm{T}$.

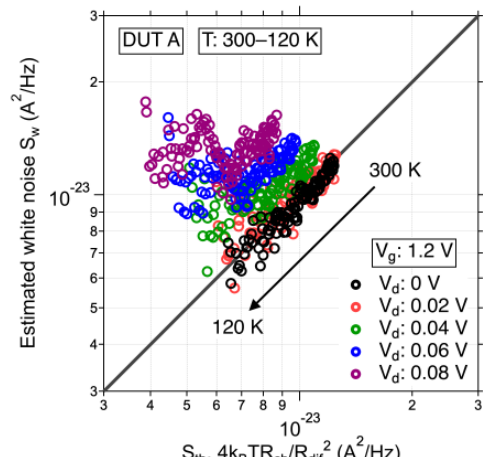

Fig. 9. Correlation between $S_{I d}$ and thermal noise $\mathrm{S}_{\text {th }}\left(4 \mathrm{k}_{\mathrm{B}} \mathrm{TR}_{\mathrm{ch}} / \mathrm{R}_{\text {dif }}^{2}\right)$ at $\mathrm{V}_{\mathrm{g}}=1.2 \mathrm{~V}$ for $\mathrm{V}_{\mathrm{d}}$ ranging from 0 to $0.08 \mathrm{~V}$. 